\title{
Broadband Variable Chromatic Dispersion in Photonic-Band Electro-Optic Waveguide
}

\author{
Kensuke Ogawa, Kozue Tomiyama and Yong Tsong Tan \\ Bussan Nanotech Research Institute Inc., Mitsui \& Co. Nanotech Park, 2-1 Koyadai, Tsukuba 305-0074, Japan \\ ke-ogawa@xnri.com \\ My The Doan, Yu Ming Bin and Dim-Lee Kwong \\ Institute of Microelectronics, 11 Science Park Road, Singapore Science Park II, Singapore 117685
}

Shigeki Yamada

Faculty of Science, Graduate School of Integrate Science, Yokohama City University, Kanazawa-Ku, Yokohama 236-0027, Japan

James B. Cole

Institute of Information Sciences and Electronics, University of Tsukuba, 1-1-1 Tennodai, Tsukuba 305-8573, Tsukuba, Japan

Yoshifumi Katayama

Tsukuba Industrial Liaison and Cooperative Research Center, University of Tsukuba, 1-1-1 Tennodai, Tsukuba 305-8577, Japan

Hiroshi Mizuta and Shunri Oda

Department of Physical Electronics, Tokyo Institute of Technology, O-okayama, Meguro-Ku, Tokyo 152-8552, Japan

\begin{abstract}
Broadband variable chromatic dispersion in C-band frequency range is demonstrated using novel nanostructured electro-optic waveguide consisting of low-loss $\mathrm{Si}_{3} \mathrm{~N}_{4}$ rectangular core and $\mathrm{Si} / \mathrm{SiO}_{2}$ two-dimensional photonic-crystal layer controlled by voltage bias.

(C)2006 Optical Society of America

OCIS codes:
\end{abstract}

\section{Introduction}

Technology for chromatic dispersion compensation is widely interested in high-speed optical-fiber communications, since chromatic dispersion plays a significant role in the propagation of short optical pulses in optical fibers [1]. Chromatic dispersion compensators have been developed using optical components such as fiber Bragg gratings, optical filters, and dispersive optics [2-4]. These compensators are designed and utilized as discrete modules installed in line in the optical fiber communication systems. With the advent of the dense wavelength-division multiplexing, increase in the number of modules becomes an issue in system construction, and there is growing demand for compact and integrated devices, which allow smaller unit sizes, lower power consumption and lower costs.

In this paper, we study the voltage dependence of chromatic dispersion in C-band frequency range using photonic-band electro-optic waveguides as potential compact chromatic dispersion compensators. We present the first demonstration of electro-optic control of chromatic dispersion in Si-based photonic-band structure.

\section{Photonic-band electro-optic waveguide}

Photonic-band electro-optic waveguides studied here were fabricated on silicon-on-insulator (SOI) wafers by deep-UV lithography and CMOS-based device processes. The main part of the waveguide is low-loss $\mathrm{Si}_{3} \mathrm{~N}_{4}$ rectangular core ( $1 \mu \mathrm{m}$ width and $400 \mathrm{~nm}$ thickness) and $\mathrm{Si} \mathrm{SiO}_{2}$ photonic crystal (PC) layer underlying the core as shown in Fig. 1. PC consists of two-dimensional triangular lattice of $\mathrm{SiO}_{2}$ pillars embedded in host Si layer. Unit cell of the triangular lattice has a pitch of $\sim 400 \mathrm{~nm}$. The diameter of $\mathrm{SiO}_{2}$ pillar is $240 \mathrm{~nm}$ or $260 \mathrm{~nm}$ in the present study. Thin SOI layer (100nm thickness) serves as host medium for the PC layer, and buried oxide layer (1 $\mu \mathrm{m}$ thickness) in SOI wafer as bottom cladding, respectively.

Refractive index of the host $\mathrm{Si}$ is controlled by voltage bias based on free-carrier absorption to realize voltage control of photonic bands. In free-carrier absorption, refractive index of Si increases with decreasing carrier concentration, and vice versa [5]. To change carrier concentration in the host $\mathrm{Si}$, the host medium was boron-doped and voltage bias is applied between drain and source electrodes illustrated in Fig. 1. High-field transport of carriers in Si produces spatial modulation of carrier concentration, known as the formation of dipole region [6]. Hole concentration in the host p-type Si is controlled by drain-source voltage.

The rectangular core of a photonic-band electro-optic waveguide governs characteristics such as mode confinement and modal loss. Modal loss is minimized by the design of the core. The PC layer, on the other hand, governs characteristics of optical phase such as chromatic dispersion. Mode field extends across the core and the PC layer, both of which form a waveguide channel. Chromatic dispersion is easily optimized in a broad spectral range with other parameters such as modal loss kept unchanged. This is a significant advantage over photonic-gap defect 
waveguides [7]. In photonic-gap defect waveguides, lightwaves are propagated as localized modes in photonic bandgap. Structure optimization for the chromatic dispersion of localized modes produces side effects such as frequency change in the localized modes themselves.

(a)

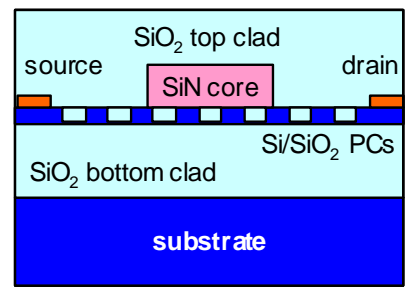

(b)

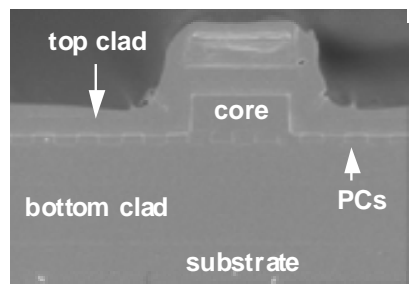

(c)

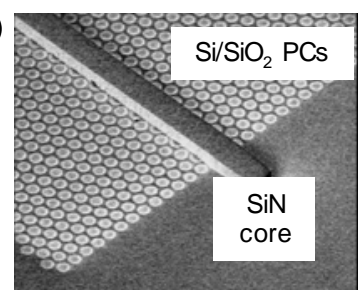

Fig. 1. (a) Schematic cross-section, (b) SEM cross-section and (c) SEM angled top view of a photonic-band electro-optic waveguide.

Dispersion curves of the 1st and 2nd bands of a photonic-band waveguide with 260nm pillar diameter were calculated by plane-wave expansion as shown in Fig. 2(a) using a slab waveguide model. The slab consists of $\mathrm{Si}_{3} \mathrm{~N}_{4}$ core layer and PC layer sandwiched between $\mathrm{SiO}_{2}$ cladding layers. The bands form guided modes if they appear below the clad lightline. Frequency of incident light, $192-196 \mathrm{THz}$ for chromatic dispersion characterization, is indicated as $v_{0}$. Only guided at $v_{0}$ is the 1 st band, and hence, the waveguide acts as a single-mode waveguide. The slope of 1st band decreases as frequency becomes closer to the lower-frequency edge of the photonic bandgap. Chromatic dispersion of the 1st band is positive and increases toward the edge of the photonic bandgap.

Spectral phase was calculated for the photonic-band waveguide by three-dimensional finite element method (3D-FEM) as plotted in Fig. 2(b). The length of the waveguide is $2.8 \mu \mathrm{m}$. The spectral phase is dominated by quadratic chromatic dispersion. The calculated spectral phase (open circles) is fitted with a parabolic curve (blue curve) with a second-order coefficient of $0.0058 \mathrm{ps}^{2}$, which leads to dispersion parameter $D=-32 \mathrm{ps} / \mathrm{nm} / \mathrm{cm}$.
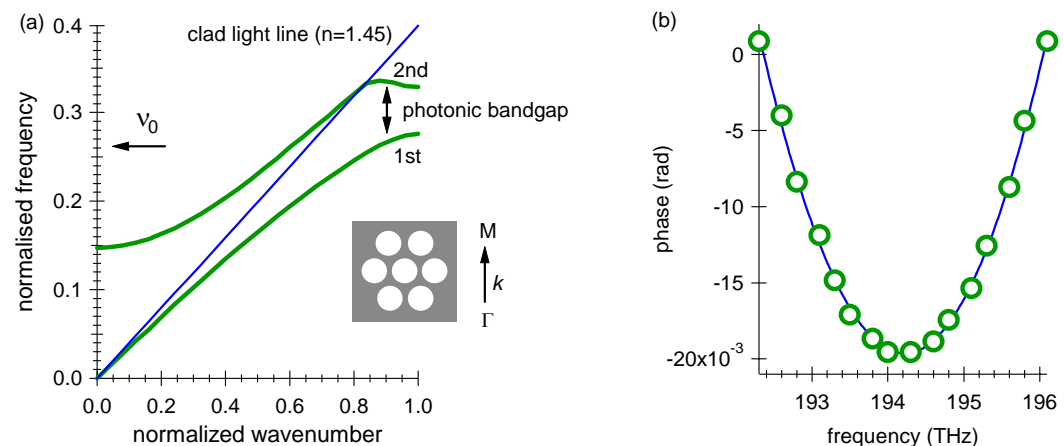

Fig. 2. (a) Calculated dispersion curves and (b) calculated spectral phase of a photonic-band electro-optic waveguide.

\section{Optical characteristics of photonic-band electro-optic waveguide}

\subsection{Fundamental characteristics}

To extract fundamental optical characteristics of the photonic band, transmission spectrum of the photonic-band waveguide with $260 \mathrm{~nm}$ pillar diameter was measured and compared with that of a rectangular $\mathrm{Si}_{3} \mathrm{~N}_{4}$ waveguide on plain SOI layer as presented in Fig. 3(a). The structure of the latter waveguide is the same as the former except that the latter contains no $\mathrm{SiO}_{2}$ pillars in $\mathrm{SOI}$ layer. The length is $1.7 \mathrm{~mm}$ for the both waveguides. Transmittance of the photonic-band waveguide decreases by more than $30 \mathrm{~dB}$ with frequency toward $199.7 \mathrm{THz}$, while that of the SOI waveguide remains nearly flat over the entire frequency range of the spectrum. The decrease is caused by the photonic band gap existing at frequencies above 199.7THz. The photonic-band (PB) edge is indicated with an arrow. Transmittance below the PB edge is associated with the 1st photonic band. Transmittance of the photonic-band waveguide is flat and about $-26 \mathrm{~dB}$ at frequencies lower than $190 \mathrm{THz}$. Most of transmission loss is produced by the optical coupling between input/output optical fibers and the waveguide. Transmission loss by the PCs is $5 \mathrm{~dB}$ or less, even though the waveguide contains nearly 2400 PC unit cells along the propagation direction. The loss will be further reduced by optimizing the fabrication processes of PCs.

Spectral phase of the photonic-band waveguide was measured in the C-band range and compared with that of the waveguide containing no PCs as in Fig. 3(b). The data were acquired by high-stability heterodyne spectral interferometry [8]. The PCs produces distinct quadratic chromatic dispersion with a positive coefficient of $4.4 \mathrm{ps}^{2}$. 
Dispersion parameter $D=41 \mathrm{ps} / \mathrm{nm} / \mathrm{cm}$ is obtained from the coefficient. This value is in good agreement with the 3D-FEM result.
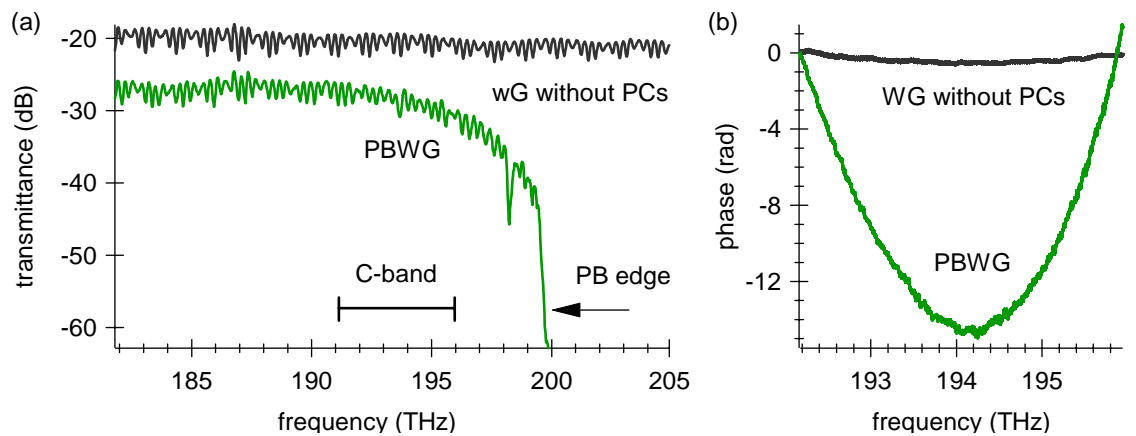

Fig. 3. (a) Transmission spectrum and (b) spectral phase of a photonic-band electro-optic waveguide (PBWG) in comparison with a waveguide which contains no PCs (WG without PCs).

\subsection{Electro-optic characteristics and variable chromatic dispersion}

The voltage dependence of transmission spectrum and spectral phase of a photonic-band waveguide with 240nm pillar diameter was studied. The measurement data are presented in Fig. 4. The photonic bandedge is shifted by $0.22 \mathrm{THz}$ to lower frequency at drain-source voltage $V_{\mathrm{DS}}=-35 \mathrm{~V}$. This shift is caused by refractive-index increase in the host $\mathrm{Si}$ layer. The refractive-index increase is originated from the decrease in free-carrier absorption [5], since the net carrier concentration in the host $\mathrm{Si}$ is decreased under the dipole-region formation [6].

Small shift $(-0.1 \%)$ in the photonic bandedge frequency causes a significant change in spectral phase and its curvature (chromatic dispersion). The coefficient of the quadratic dispersion term at $V_{\mathrm{DS}}=0 \mathrm{~V}$ is obtained as $5.7 \mathrm{ps}^{2}$ from curve fitting, and $D=-53 \mathrm{ps} / \mathrm{nm} / \mathrm{cm}$. The quadratic-term coefficient is increased $5.8 \%$ at $V_{\mathrm{DS}}=-35 \mathrm{~V}$, and dispersion parameter as $-56 \mathrm{ps} / \mathrm{nm} / \mathrm{cm}$. This increase is a characteristic of the electro-optic effect on the 1st photonic band since the chromatic dispersion of the 1st band becomes larger with increasing frequency toward the photonic bandedge as expected from the dispersion curve in Fig. 2(a). This is the first experimental evidence for broadband variable chromatic dispersion in a Si-based nanostructured electro-optic waveguide.
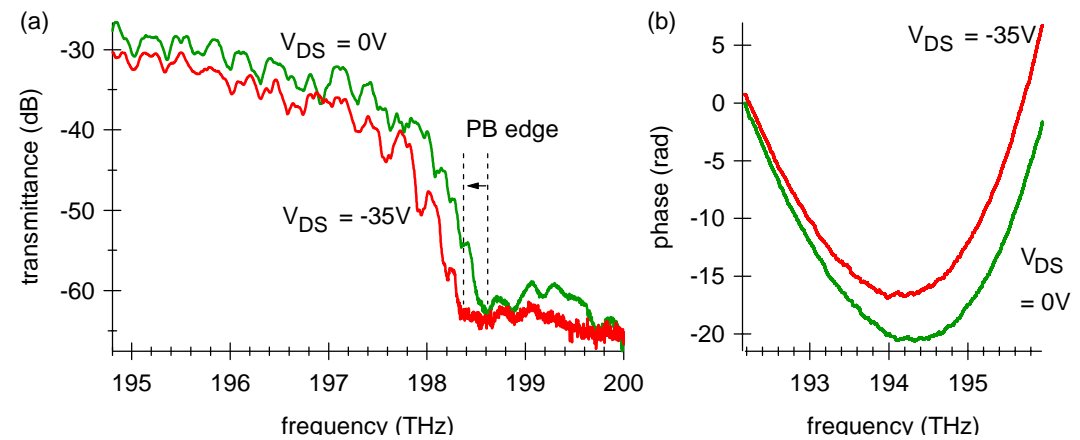

Fig. 4. (a) Voltage bias dependence of transmission spectrum and (b) spectral phase of a photonic-band electro-optic waveguide.

\section{References}

[1] Allan E. Willner and Bogdan Hoanca, "Fixed and tunable management of fiber chromatic dispersion," in Optical fiber telecommunications IV-B: systems and impairments, I. P. Kaminow and T. Li, ed. (Academic, 2002) Chap. 14.

[2] B. J. Eggleton et al, "Integrated tunable fiber gratings for dispersion management in high bit rate systems," J. Lightwave Technol. 18, 1418-1432 (2000).

[3] C. K. Madsen et al, "Integrated all-pass filters for tunable dispersion and dispersion slope compensation,” IEEE Photonics Technol. Lett. 11, 1623-1625 (1999).

[4] M. Shirasaki, “Chromatic dispersion compensator using virtually imaged phased array,” IEEE Photonics Technol. Lett. 9, 1598-1600 (1997).

[5] R. A. Soref et al, “Electrooptical effects in silicon,” IEEE J. Quantum Electron. QE-23, 123-129 (1987).

[6] K. Lehovec, "Field distribution in junction field-effect transistors at large drain voltages,” IEEE Trans. Electron Devices ED-22, 273-281 (1975).

[7] M. Notomi et al, "Extremely large group-velocity dispersion of line-defect waveguides in photonic crystal slabs,” Phys. Rev. Lett. 87, 253902-1-4 (2001).

[8] K. Ogawa and T Lay, "Chromatic dispersion measurement of SOA in C + L band by self-tracking real-time interferometry," 2005 Optical Fiber Communication Conference Technical Digest (2005) OThH7. 